\title{
COVID-19 pandemia and inherited cardiomyopathies and channelopathies: a short term and long term perspective
}

\author{
Giuseppe Limongelli ${ }^{1,2,3,4^{*}}$ and Lia Crotti ${ }^{4,5,6,7}$
}

\begin{abstract}
Inherited heart disease represent a very heterogenous group of cardiac disorders, characterized by inherited, acquired, and often rare disorders affecting the heart muscle (cardiomyopathies) or the cardiac electrical system (ion channel disease). They are often familial diseases, and are among the leading cause of juvenile sudden death and heart failure. The aim of this paper is to give a perspective on how to run a clinical service during an epidemic or pandemic emergency and to describe the potential COVID-19 associated risks for patients affected by inherited heart diseases.
\end{abstract}

Keywords: Inherited and rare heart disease, Cardiomyopathies, Channellopathies, COVID-19

\section{Introduction}

The severe acute respiratory syndrome coronavirus-2 (SARS-Cov-2) has been recently declared as pandemic by $\mathrm{WHO}$, emerging as a global threat due to the high transmission rate of the virus. The infection may have a wide range of clinical manifestation from no symptoms to interstitial pneumonia, acute respiratory distress and/ or systemic disease. Moreover, data show that preexisting cardiovascular diseases may favor more severe clinical manifestations [1], and conversely SARS-CoV-2 may cause myocardial injury [2]. The exponential need of hospitalization and intensive care due to COVID-19 infections thoroughly changed priorities of healthcare systems around the world. Hospitals were in part or completely dedicated to Covid-19 patients and therefore routine management of patients with inherited

\footnotetext{
* Correspondence: limongelligiuseppe@libero.it

'Inherited and Rare Cardiovascular Disease Clinic, Department of

Translational Medical Sciences, University of Campania "Luigi Vanvitelli", Via L. Bianchi 1 c/o Monaldi Hospital, AORN Colli, Naples, Italy

${ }^{2}$ Institute of Cardiovascular Sciences, University College of London and St. Bartholomew's Hospital, London, UK

Full list of author information is available at the end of the article
}

channelopathies and cardiomyopathies was suspended in most hospitals.

Inherited and rare heart diseases represent a very heterogenous group of congenital, inherited or acquired disorders affecting the heart muscle (cardiomyopathies) or the electrical system (channelopathies). They are often familial diseases, and are among the leading cause of juvenile sudden death and heart failure. The aim of this paper is to give a perspective on how to run an inherited and rare disease clinical service during an epidemic or pandemic emergency and to describe the potential COVID-19 associated risks for patients affected by these diseases.

\section{What are the risks associated with Covid-19 infection for patients with inherited cardiomyopathies and channelopathies?}

Channelopathies are a group of genetically transmitted heart diseases, in which the heart is mainly structural normal, but there is an increased risk of life-threatening arrhythmias.

In Long QT Syndrome (LQTS) [3], QT prolonging drugs should be avoided as they could favor torsades 
de pointes (TdP) and sudden cardiac death [4]. In case of COVID-19 infection most of the drugs currently used (Table 1) can prolong QT interval $[5,6]$ and some of them may cause diarrhea, favoring hypokalemia, another factor increasing arrhythmia risk [3]. Furthermore, diarrhea is one of the clinical manifestation of COVID-19 infection (Table 2) [1]. Therefore, specific caution should be suggested in LQTS patients to avoid infection and whenever they get it is, it is clearly dangerous an in house management with drugs in the absence of an adequate QT monitoring. Whenever an in-hospital admission is needed, a careful QT monitoring and a telemetric system should be used. A careful balance of pros and cons should guide the decision to discontinue therapy in case of important QT prolongation. Furthermore, in the absence of clear benefit and safety data from well designed, randomized, controlled clinical trials, therapies associated with greater QT prolongation and arrhythmic risk, as hydroxychloroquine/chloriquine particularly if in association with macrolide (azitromicin), should be avoided. Mandatory is going on with beta-blocker therapy and keep potassium level above $4 \mathrm{mEq} / \mathrm{l}$ with potassium supplements.

Very recently, CredibleMeds launched an important new decision support program to help clinicians manage the risk of QT prolonging medications when treating patients with COVID-19. Accessing at MedSafety Scan ${ }^{\circ}$ (https://medsafetyscan.org) and entering each patient's clinical risk factors and their drugs, the program quickly reports if any of the medicines are on the QTdrugs lists and calculates the patient's QTscore for risk of QT prolongation and torsades.

In Short QT Syndrome (SQTS) [3], there is no particular concern related to COVID-19 infection, the only precaution should be avoid hypokalemia that is a proarrhythmic factor. The same apply to catecholaminergic polymorphic ventricular tachycardia (CPVT), in which an additional risk could be the use of alpha or beta adrenergic mimetic drugs in case a hemodynamic support is needed [6].

For Brugada Syndrome fever related to infection is the real danger and a more careful and tailored evaluation of criteria for admission to Hospital should be applied [6].

Cardiomyopathies (CMPs) represent an heterogeneous group of inherited disease of the heart muscle, characterized by different phenotypes (hypertrophic, HCM; dilated, DCM; arrhythmogenic, AC; restrictive, RCM) and dysfunction (diastolic and/or systolic), with an increased risk of life-threatening arrhythmias and heart failure with preserved or reduced ejection fraction (HFpEF or HFrEF).

It is now clear that Covid-19 infection has a wide spectrum of presentation and complications, related to the virus itself or secondary to the inflammatory and immune response [7]. Myocardial injury, evidenced by elevated high-sensitivity troponin levels, is more frequently observed in severe cases, and it is associated with the higher inflammatory burden that can induce vascular inflammation, myocarditis, and cardiac arrhythmias [8].

Covid-19 infection in patients with CMPs represent a concrete risk of worsening patient clinical status, particularly in those who experienced previous HF events or with end stage disease. Moreover, the combination of hypokalemia and prolonged QT exponentially increases the risk of arrhythmias. Hospitalization should be considered, after teleconsultation, in patients with moderate-high risk of disease worsening.

Also, there is a specific risk related to CMP phenotype (i.e. the risk of dehydration can be much higher in patients with obstructive vs non obstructive HCM) [9] or etiology (increase risk of metabolic decompensation or lactic acidosis in patients with metabolic and mitochondrial CMPs and myopathies) (Table 3) $[10,11]$.

\section{How to run an inherited heart disease clinic during COVID-19 pandemia}

The big challenge during the pandemic is the "list of priorities" that the healthcare system need to redesign to face the emergency. The healthcare systems of the vast majority of western countries have been built to treat cardiovascular and neurological emergencies, and to treat chronic patients with different disease. In this latter, inherited and rare disease represent a wide spectrum of cardiovascular disease generally diagnosed and managed by a multidisciplinary setting of expert physicians. As in Fig. 1, we designed a 5 levels list of priorities in these patients: 1. Level 1: all the patients with severe, new onset symptoms (heart failure, HF) or lifethreatening arrhythmias requiring immediate treatment; pregnancy management; nondeferrable surgical or percutaneous treatments; 2. Level 2: urgent diagnostic, devices, percutaneous, or surgical procedures; 3. Level 3: new symptoms onset or progressive symptomatology; new referral; 4. Level 4: patients follow-up; 5. Level 5: family screening. The first 2 levels deserve immediate or urgent hospitalization for procedures or nondeferrable treatments. This should follow a "non COVID-19 pathway", with dedicated spaces and equipment. Before admission, or at admission for very urgent condition, a COVID-19 PCR nasal and throat swab should be performed in all patients. The 3rd and 4rth level can be managed with teleconsultation, eventually followed by outpatient clinic admission. Family screening can be planned for the post-emergency phase (phase 2).

Phase 2 is the most challenging phase. Since phase 1 had a "leopard spot pattern" of presentation, progression and severity in different regions and countries, timing 


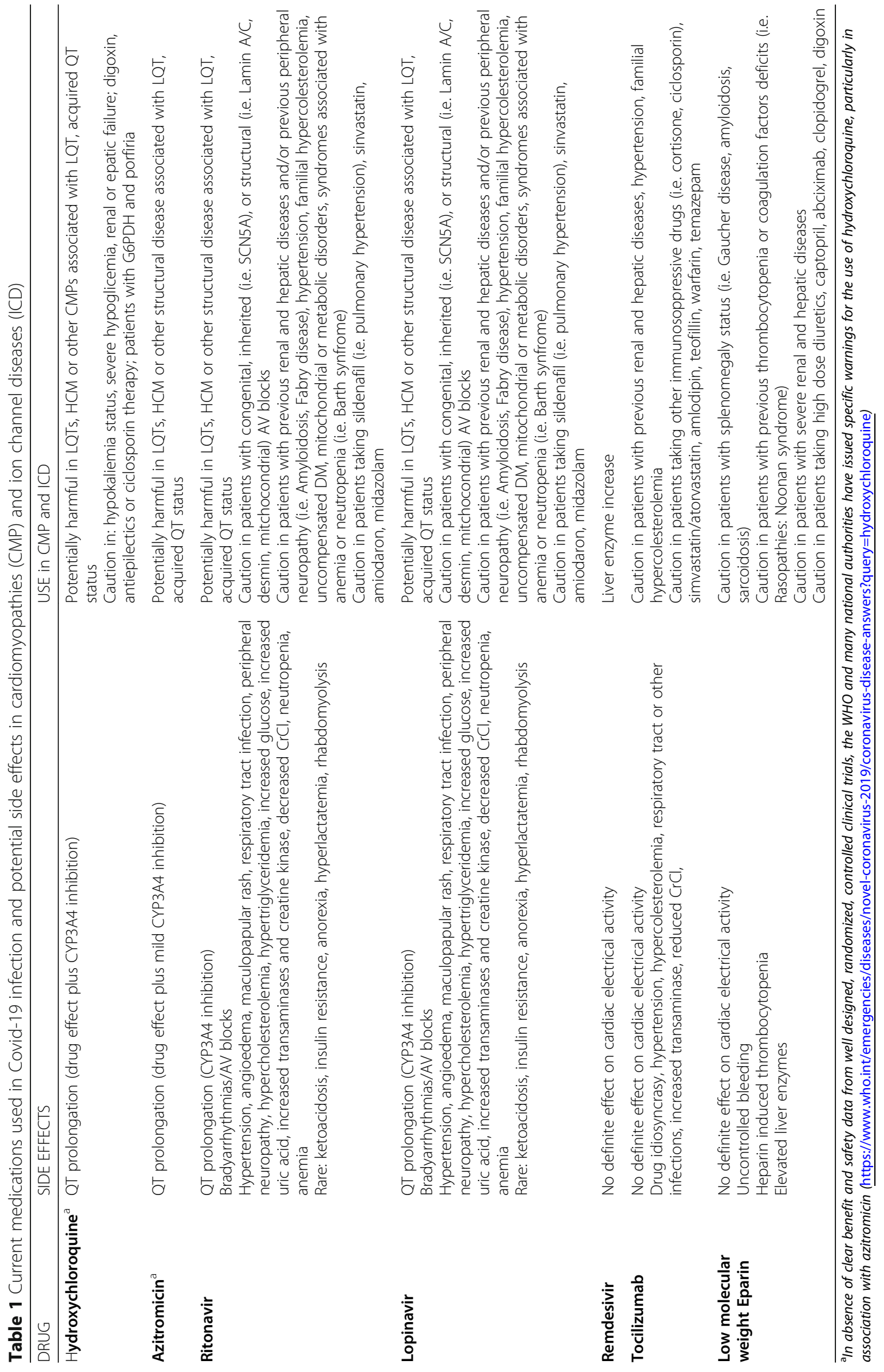




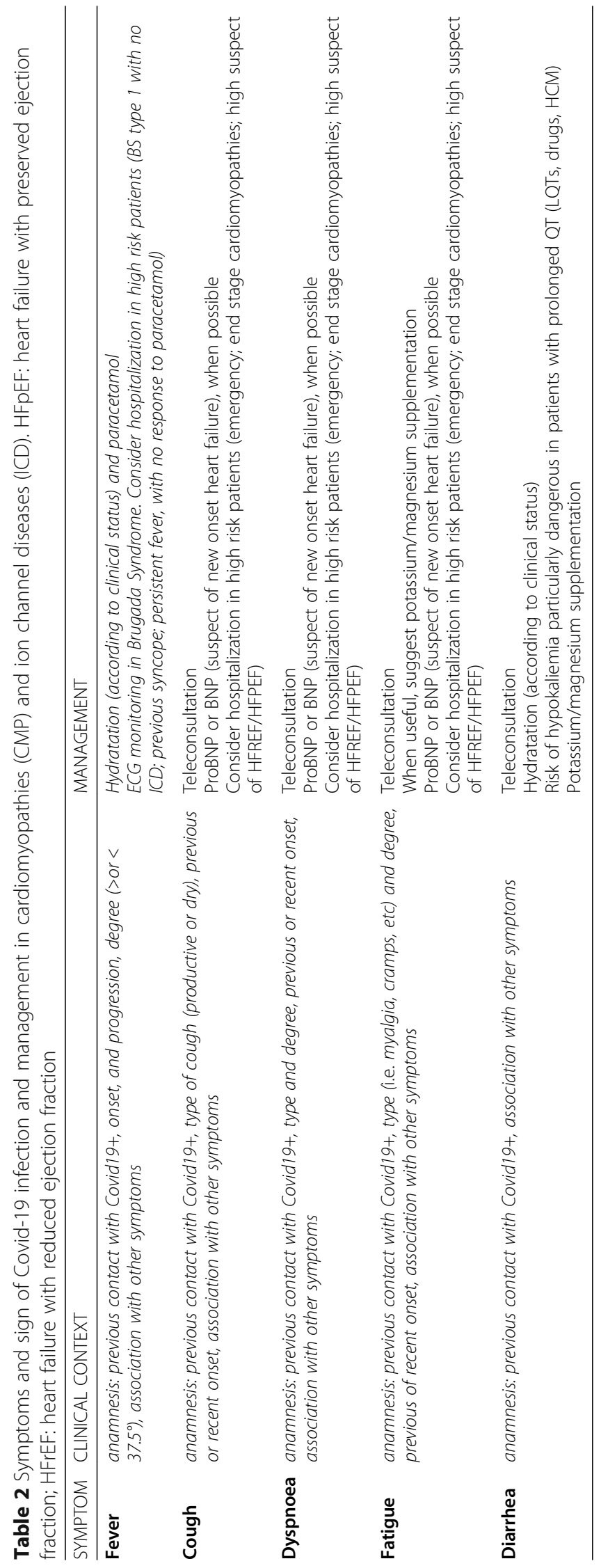




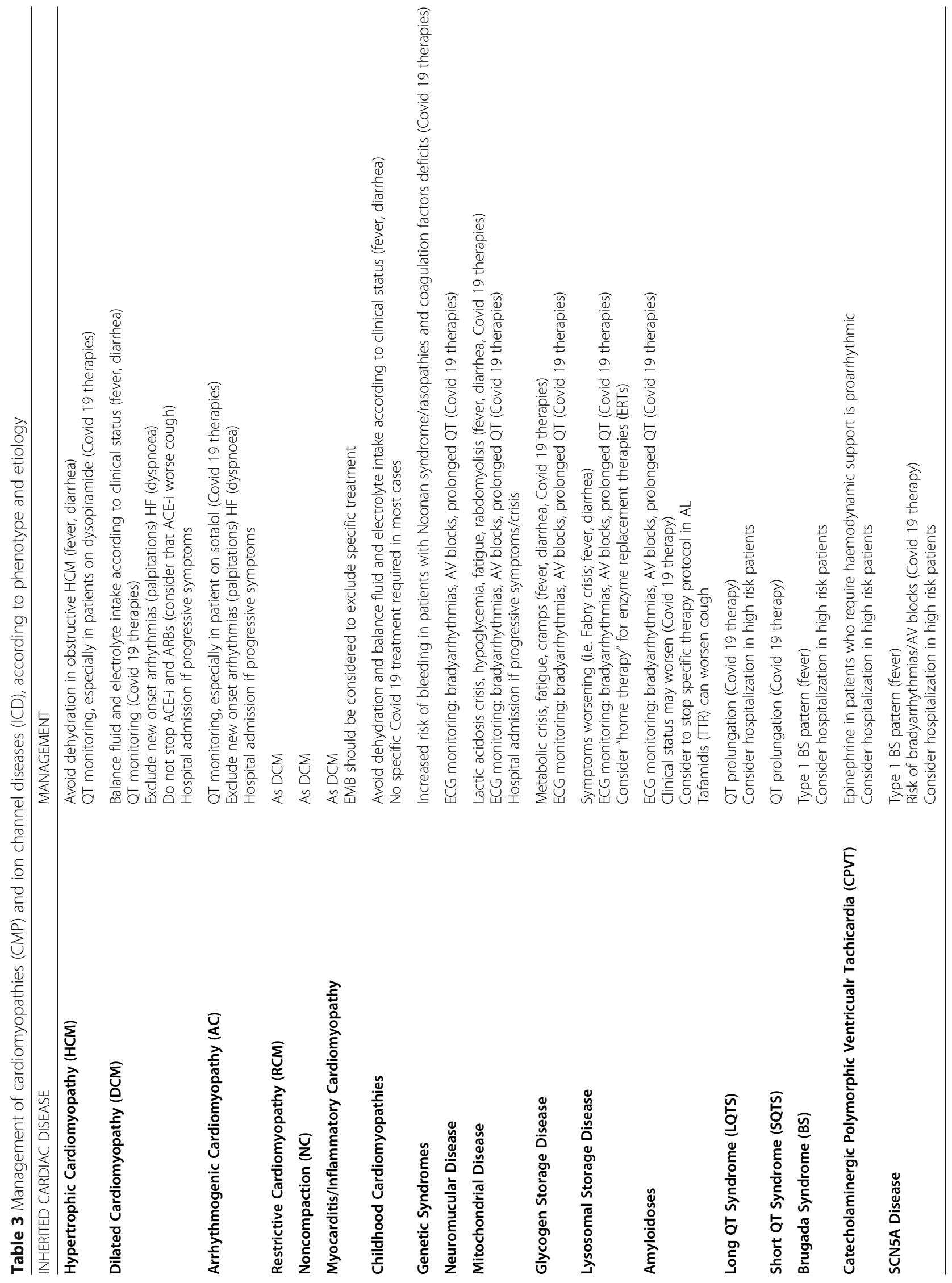




\begin{tabular}{|c|c|}
\hline $\begin{array}{l}\text { Device implantation in inherited and rare heart disease } \\
\text { (secondary prevention) }\end{array}$ & Hospital Admission (inpatient) - procedure \\
\hline $\begin{array}{c}\text { New onset heart failure or life-threatening arrhythmias in inherited and } \\
\text { rare heart disease }\end{array}$ & Hospital Admission (inpatient) - specific treatment \\
\hline Pregnancy in inherited and rare heart disease & Hospital (inpatient) Admission - delivery \\
\hline Nondeferrable surgical or percutaneous treatments & Hospital (inpatient) Admission - procedure \\
\hline $\begin{array}{l}\text { Device Implantation in inherited and rare heart disease } \\
\text { (primary prevention) }\end{array}$ & Hospital (inpatient) Admission - procedure \\
\hline Surgical or percutaneous treatments & Hospital (inpatient) Admission - procedure \\
\hline Diagnostic procedures (right cath, coronary angiography, RF ablation) & Hospital (inpatient) Admission - procedure \\
\hline Unexplained syncope in inherited and rare heart disease & Hospital (outpatient) Admission - specific treatment \\
\hline New symptoms onset in inherited and rare heart disease & Telemedicine -> Hospital (outpatient) Admission \\
\hline Progression of symptoms in inherited and rare heart disease & Telemedicine -> Hospital (outpatient) Admission \\
\hline New referral patient (suspect) & Telemedicine $->$ Hospital (outpatient) Admission \\
\hline Follow-up & $\begin{array}{c}\text { Telemedicine }>\text { Hospital (outpatient) Admission or } \\
\text { waiting list (Phase 2) }\end{array}$ \\
\hline Family Screening & Waiting List (Phase 2) \\
\hline
\end{tabular}

Fig. 1 how to run an inherited heart disease clinic during covid-19 pandemia. List of priorities

and specific measure for phase 2 can be different and region/country specific. Nevertheless, there is a common goal that should be pursued, i.e. population safety and the avoidance of virus spreading. This could be obtained considering anyone as potentially positive. Therefore, for everyone, social distancing and the use of facial masks should be mandatory and adequate DPI should be worn by healthcare personnel, to avoid not only that they get ill but that they could become carriers of the infection. Population control with the use of COVID-19 IgG/IgM (and, in selected cases, COVID-19 PCR nasal and throat swab) associated with smart-phone technologies, could be of great help to guide a safer phase 2 .

In term of organization of the inherited heart disease clinic, 4 main points should be considered:

1. a priority based approach: different levels of intervention (inhospital admission, outpatient clinic evaluation, teleconsulting) should be created according to the clinical status and specific needs of different patients. The same applies for hospital based and/or "home therapies" (as for patients with CMPs and lysosomal storage disorders).
2. triage step: a team of doctors, nurses, and trainees, should be in charge for the triage step, i.e. to define a new agenda of the outpatient clinic which is priority based.

3. teleconsultation/telemedicine: before admission, the triage team can preventively contact by teleconsulting/ telemedicine platforms patients and their families, to collect past and recent clinical history (including, any symptoms or contact with infected individuals).

4. safety procedures: an online booking and payment should be provided by the hospital/academic organization. The admission in the outpatients clinic will be preordered to avoid crowded waiting rooms and favor social distancing, as much as possible. Adult patients will be invited to come alone (with very few exceptions) to clinic, while no more than 1 person will be allowed to come with children. At admission, body temperature will be measured, and surgical masks should be worn by the all patients.

\section{Conclusions}

Patients with inherited CMPs and channelopaties are at potential higher risk during a COVID-19 infection and 
disease-specific recommendation and precaution should be employed. There are risks associated with the infection itself, which can cause interstitial pneumonia, but could also have a cardiac and/or systemic involvement, and risks related to COVID-19 treatment. Moreover, litthe is known about medium- and long-term consequences of this infection that could theoretically favor CMPs secondary to acute cardiac injuries (i.e. myocarditis, acute coronary syndromes) or to pulmonary sequelae that may favor chronic pulmonary heart.

Inherited and rare disease clinical services should adjust their way of managing patients during an epidemic or pandemic emergency and we proposed possible schemes. A teleconsulting/telemedicine approach should be favorably deemed during Covid-19 emergency, and should strongly support accomplishment of phase 2 .

\section{Abbreviations}

AC: Arrhythmogenic cardiomyopathy; CMPs: Cardiomyopathies; CPVT: Catecholaminergic polymorphic ventricular tachycardia; DCM: Dilated cardiomyopathy; HFpEF: Heart failure with preserved ejection fraction; HFrEF: Heart failure with reduced ejection fraction; HCM: Hypertrophic cardiomyopathy; LQTS: Long QT Syndrome; RCM: Restrictive cardiomyopathy; SARS-Cov-2: Severe acute respiratory syndrome coronavirus-2; SQTS: Short QT Syndrome

\section{Acknowledgements}

We are grateful to all the colleagues (doctors and nurses) working in our Units for the great effort spent during COVID 19 emergency.

\section{Authors' contributions}

G.L. conceived the study. G.L. and L.C. wrote, edited, and approved the final version of the manuscript.

\section{Funding}

This research received no specific grant from any funding agency in the public, commercial or not-for-profit sectors.

\author{
Availability of data and materials \\ N/A
}

\section{Ethics approval and consent to participate}

N/A

\section{Consent for publication}

Participants provided written informed consent.

\section{Competing interests}

The authors declare that they have no competing interests.

\section{Author details}

IInherited and Rare Cardiovascular Disease Clinic, Department of Translational Medical Sciences, University of Campania "Luigi Vanvitelli", Via L. Bianchi 1 c/o Monaldi Hospital, AORN Colli, Naples, Italy. Institute of Cardiovascular Sciences, University College of London and St. Bartholomew's Hospital, London, UK. ${ }^{3}$ Centro coordinamento malattie rare regione campania - via L. bianchi 1 c/o monaldi hospital - ao colli - Naples, Naples, Italy. ${ }^{4}$ European Reference Network for Rare and Complex Diseases of the Hearthttp://guardheart.ern-net.eu. ${ }^{5}$ Istituto Auxologico Italiano, IRCCS, Department of Cardiovascular, Neural and Metabolic 5. Sciences, San Luca Hospital, Milan, Italy. ${ }^{6}$ Istituto Auxologico Italiano, IRCCS, Center for Cardiac Arrhythmias of Genetic Origin, Laboratory of Cardiovascular Genetics, Milan, Italy. ${ }^{7}$ Department of Medicine and Surgery, University of Milano-Bicocca, Milan, Italy.
Received: 27 April 2020 Accepted: 16 June 2020

Published online: 22 June 2020

\section{References}

1. Wang D, Hu B, Hu C, Zhu F, Liu X, Zhang J, Wang B, Xiang H, Cheng Z, Xiong Y, Zhao Y, Li Y, Wang X, Peng Z, et al. JAMA. 2020. https://doi.org/10. 1001/jama.2020.1585.

2. Zheng YY, Ma YT, Zhang JY, Xie X. COVID-19 and the cardiovascular system. Nat Rev Cardiol. 2020;17(5):259-60. https://doi.org/10.1038/s41569-020-0360-5.

3. Crotti L, Odening KE, Sanguinetti MC. Heritable arrhythmias associated with abnormal function of cardiac potassium channels. Cardiovasc Res. 2020. https://doi.org/10.1093/cvr/cvaa068 [Epub ahead of print].

4. Schwartz PJ, Woosley RL, Crotti L. When prescribing drugs, do medical doctors and healthcare professionals realize that their patient has the long QT syndrome? Eur Heart J. 2019;40(37):3118-20. https://doi.org/10.1093/ eurheartj/ehz355.

5. Giudicessi JR, Noseworthy PA, Friedman PA, Ackerman MJ. Urgent guidance for navigating and circumventing the QTc prolonging and torsadogenic potential of possible pharmacotherapies for COVID-19. Mayo Clin Proc. 2020;95(6):1213-21. https://doi.org/10.1016/j.mayocp.2020.03.024 Epub 2020 Apr 7.

6. Wu Cl, Postema PG, Arbelo E, Behr ER, Bezzina CR, Napolitano C, Robyns T, Probst V, Schulze-Bahr E, Remme CA, Wilde AAM. SARS-CoV-2, COVID-19 and inherited arrhythmia syndromes. Heart Rhythm. 2020;51547-5271(20): 30285. https://doi.org/10.1016/j.hrthm.2020.03.024.

7. Libby P. The heart in COVID19: primary target or secondary bystander?JACC basic Transl Sci; 2020. https://doi.org/10.1016/j.jacbts.2020.04.001.

8. Madjid M, Safavi-Naeini P, Solomon SD, Vardeny O. Potential effects of coronaviruses on the cardiovascular system: a review. JAMA Cardiol. 2020 https://doi.org/10.1001/jamacardio.2020.1286.

9. Pelliccia F, Alfieri O, Calabrò P, Cecchi F, Ferrazzi P, Gragnano F, Kaski JP, Limongelli G, Maron M, Rapezzi C, Seggewiss H, Yacoub MH, Olivotto I. Multidisciplinary evaluation and management of obstructive hypertrophic cardiomyopathy in 2020: towards the HCM heart team. Int J Cardiol. 2020; 304:86-92. https://doi.org/10.1016/.i.jcard.2020.01.021.

10. Limongelli G, Monda E, Tramonte S, Gragnano F, Masarone D, Frisso G, Esposito A, Gravino R, Ammendola E, Salerno G, Rubino M, Caiazza M, Russo M, Calabrò P, Elliott PM, Pacileo G. Prevalence and clinical significance of red flags in patients with hypertrophic cardiomyopathy. Int J Cardiol. 2020; 299:186-91. https://doi.org/10.1016/j.jijcard.2019.06.073.

11. Limongelli G, Masarone D, D'Alessandro R, Elliott PM. Mitochondrial diseases and the heart: an overview of molecular basis, diagnosis, treatment and clinical course. Futur Cardiol. 2012;8(1):71-88. https:/doi.org/10.2217/fca.11.79.

\section{Publisher's Note}

Springer Nature remains neutral with regard to jurisdictional claims in published maps and institutional affiliations.
Ready to submit your research? Choose BMC and benefit from:
- fast, convenient online submission
- thorough peer review by experienced researchers in your field
- rapid publication on acceptance
- support for research data, including large and complex data types
- gold Open Access which fosters wider collaboration and increased citations
- maximum visibility for your research: over $100 \mathrm{M}$ website views per year
At $\mathrm{BMC}$, research is always in progress.
Learn more biomedcentral.com/submission 